\title{
New-onset giant cell arteritis following COVID-19 mRNA (BioNTech/ Pfizer) vaccine: a double-edged sword?
}

\author{
Ana M. Anzola ${ }^{1,2} \mathbb{D} \cdot$ Laura Trives $^{1,2} \mathbb{D} \cdot$ Julia Martínez-Barrio ${ }^{1,2} \mathbb{D} \cdot$ Blanca Pinilla $^{2,3}$ (D) José María Álvaro-Gracia ${ }^{1,2} \mathbb{D}$. \\ Juan Molina-Collada ${ }^{1,2}$ iD
}

Received: 27 October 2021 / Revised: 17 December 2021 / Accepted: 23 December 2021 / Published online: 3 February 2022

(c) International League of Associations for Rheumatology (ILAR) 2022

\section{Dear Editor,}

COVID-19 vaccination is a global effort. In Spain, over $79 \%$ of the population is fully vaccinated and, although the benefits obtained from immunization clearly outweigh any potential risks, we have noticed a few case reports of autoimmune diseases possibly triggered by the vaccine in the last year $[1,2]$.

An 83-year-old woman with controlled dyslipidemia and hypertension was admitted to our emergency department with a 2-week history of disruptive cervical pain, headache, and scalp tenderness. She did not have jaw claudication or visual manifestations and polymyalgia rheumatica symptoms were absent. Symptoms began $24 \mathrm{~h}$ after the first dose of COVID-19 mRNA (BioNTech/Pfizer) vaccine and were attributed to a post-vaccination reaction. However, the symptoms worsened during the following weeks and the case was reassessed. Tenderness on the right temporal

Juan Molina-Collada

molinacolladajuan@gmail.com

Ana M. Anzola

anamelissa89@gmail.com

Laura Trives

triveslaura@gmail.com

Julia Martínez-Barrio

juliamartinezbarrio@gmail.com

Blanca Pinilla

blanca.pinilla@salud.madrid.org

José María Álvaro-Gracia

jalvarogracia@gmail.com

1 Department of Rheumatology, Hospital General

Universitario Gregorio Marañón, Calle del Dr. Esquerdo, 46, 28007 Madrid, Spain

2 Instituto de Investigación Sanitaria Gregorio Marañón (IiSGM), Madrid, Spain

3 Department of Internal Medicine, Hospital General Universitario Gregorio Marañón, Madrid, Spain artery and a faint induration in this location were present on physical examination, but no vascular murmur was detected. The patient's vital signs were normal. Her initial blood test revealed elevated inflammatory markers (fibrinogen $>1000 \mathrm{mg} / \mathrm{dl}$, C-reactive protein $13.6 \mathrm{mg} / \mathrm{dl}$, erythrocyte sedimentation rate $71 \mathrm{~mm} / \mathrm{h}$ ). Protein chain reaction SARS-CoV2 was negative. An ultrasound exam in our fasttrack clinic showed a non-compressible halo sign in the parietal branch of the right temporal artery (Fig. 1). Large vessel examination (carotid, subclavian, and axillary) was normal. Suspecting a new-onset giant cell arteritis (GCA), pulse steroids plus methotrexate were started. An FDG-PET/ CT scan revealed an abnormal artery uptake suggestive of bilateral vertebral vasculitis, mainly on the in-bone portion of the artery (Fig. 1). Although the right temporal artery biopsy (TAB) was normal, the patient met the ACR criteria for GCA. After treatment, acute-phase reactants returned to normal and symptoms disappeared. Follow-up ultrasound 3 and 6 months later showed moderate improvement of the wall thickening. Our patient received the second vaccine dose while still on medium-dose steroids, but presented no relapse symptoms. Currently, she is in remission with weekly methotrexate and low-dose steroids.

The COVID-19 pandemic has triggered a "vaccines race" giving us the first mRNA vaccines as a result. Even though vaccination started almost a year ago, we have only begun to understand their effect on autoimmunity. mRNA vaccines take advantage of protein translation to encode viral proteins. These are presented in the major histocompatibility complex to create an immune response against the virus. It makes vaccines safer and faster to develop since no pathogens or toxins are used. Nonetheless, we are taking advantage of the same mechanisms that cause antigen cross-reactivity; therefore, there is a concern about triggering autoimmune events [3]. Several studies on adverse events have shown an overall safe profile even in patients with known immune diseases [4, 5]. However, studies are designed to identify mainly common 
Fig. 1 A Longitudinal US scan showing a hypoechoic "halo sign" of the right parietal branch of the temporal artery with increased intima media thickness (black arrow with white border). B Longitudinal and coronal PET scan showing abnormal artery uptake highly suggestive of bilateral vertebral vasculitis, mainly in the in-bone portion up to the basilar artery (white arrows)

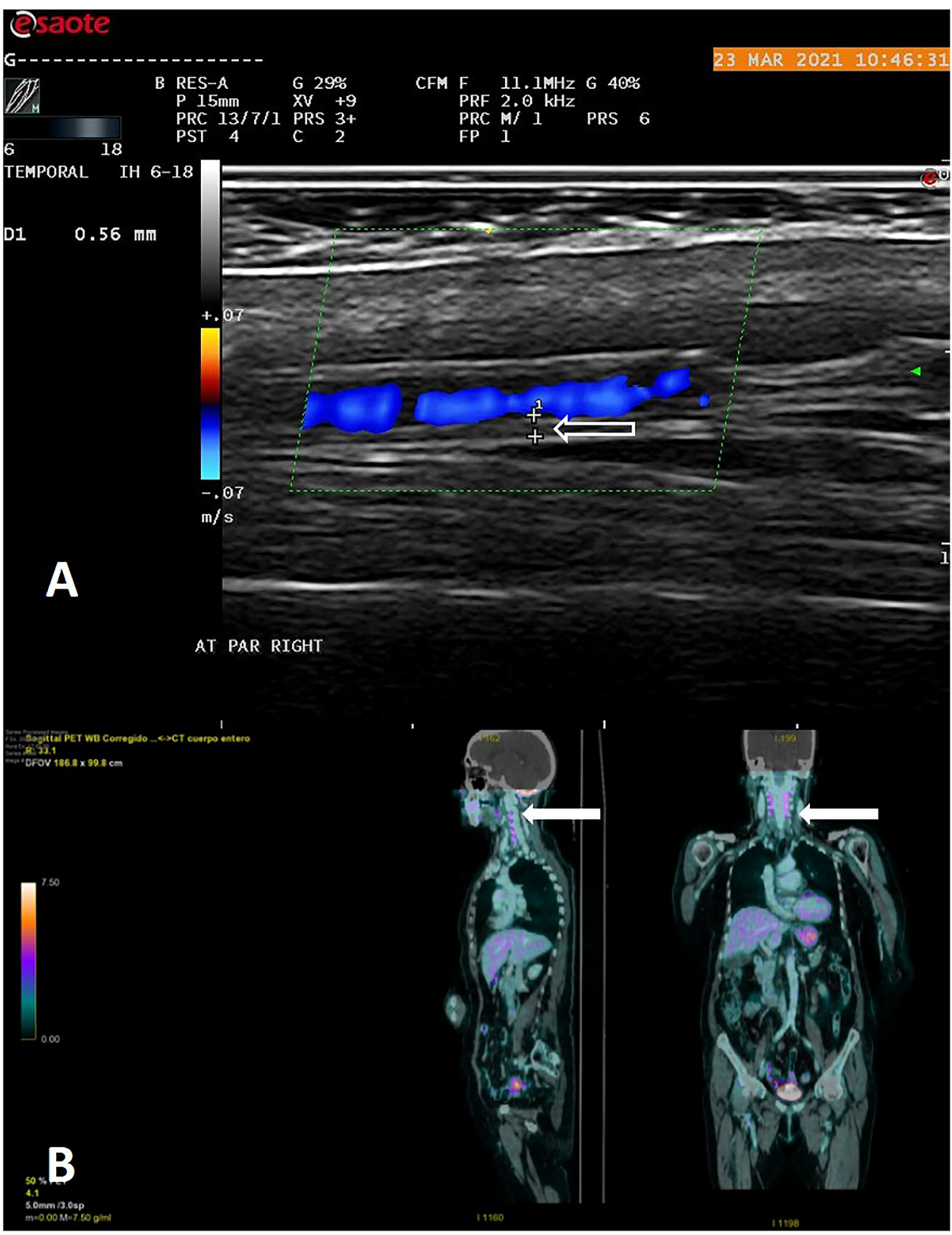

and short-term events [6]. With millions vaccinated at this moment, and vaccine booster shots being administered, it is still early to understand the long-term effects or the safety profile regarding more uncommon events. Several reports of new-onset immune events have been published $[7,8]$.

One of the hypotheses on the pathophysiology of GCA highlights the role of an infectious agent. This conjecture derives from the seasonal incidence of the disease, viral antigens on temporal artery biopsies, and several reports regarding viral entities such as varicella-zoster [9] and, more recently, SARS-COV-2 as possible GCA triggers. Likewise, a relationship with the influenza vaccine has been described [10]. In a recently published case of GCA related to the mRNA vaccine, a similar observation was made regarding these vaccines and their ability to induce cross-reactivity and trigger self-recognition using different mechanisms [2]. Although they seem to be rare events, the true scope of this issue is still uncertain. Recently, a pharmacovigilance study was published revealing a higher incidence of both giant GCA and polymyalgia rheumatica (PMR) following mRNA vaccines supporting a causal relationship [1]. Since the possibility of incidental coexistence in the present case cannot be discarded, further studies should be undertaken to better understand the potential uncommon effects of these vaccines.

We think that while the overall incidence of vaccine-triggered autoimmunity is low, vaccinations should continue 
as planned. However, rheumatologists worldwide should be aware of autoimmune diseases as a new potential adverse event of mRNA vaccines.

Author contribution -Ana M. Anzola: conception and design of study, drafting the manuscript, approval of the version of the manuscript to be published

-Laura Trives: acquisition of data, revising the manuscript critically for important intellectual content, approval of the version of the manuscript to be published

-Julia Martinez-Barrio: analysis of data, revising the manuscript critically for important intellectual content, approval of the version of the manuscript to be published

-Blanca Pinilla: acquisition of data, revising the manuscript critically for important intellectual content, approval of the version of the manuscript to be published

-José María Álvaro-Gracia: analysis and revision of data, revising the manuscript critically for important intellectual content, approval of the version of the manuscript to be published

-Juan Molina: conception and design of study, interpretation of data, drafting the manuscript, approval of the version of the manuscript to be published

Data availability Relevant data included in main text, additional data available upon request. No databases were generated. No additional data was analyzed.

\section{Declarations}

Ethics approval Patient consent for publication was obtained. The patient reviewed the final draft and images and accepted their use for publication. Consent documents are available upon request.

Editing No paid editing support was involved in the creation or edition of this article. Manuscript was reviewed for grammatical errors, language mistakes, and coherency by US-based healthcare professionals for free.

Disclosures None.

\section{References}

1. Mettler C, Jonville-Bera A-P, Grandvuillemin A et al (2021) Risk of giant cell arteritis and polymyalgia rheumatica following COVID-19 vaccination: a global pharmacovigilance study.
Rheumatology 19:82-82. https://doi.org/10.1093/rheumatology/ keab756

2. Mejren A, Sørensen CM, Gormsen LC et al (2021) Large-vessel giant cell arteritis after COVID-19 vaccine. Scand J Rheumatol 1961401:1-2. https://doi.org/10.1080/03009742.2021.1961401

3. Pardi N, Hogan MJ, Porter FW, Weissman D (2018) mRNA vaccines-a new era in vaccinology. Nat Rev Drug Discov 17:261-279. https://doi.org/10.1038/nrd.2017.243

4. Geisen UM, Berner DK, Tran F et al (2021) Immunogenicity and safety of anti-SARS-CoV-2 mRNA vaccines in patients with chronic inflammatory conditions and immunosuppressive therapy in a monocentric cohort. Ann Rheum Dis 80:1306-1311. https:// doi.org/10.1136/annrheumdis-2021-220272

5. Braun-Moscovici Y, Kaplan M, Braun M et al (2021) Disease activity and humoral response in patients with inflammatory rheumatic diseases after two doses of the Pfizer mRNA vaccine against SARS-CoV-2. Ann Rheum Dis 80:1317-1321. https://doi.org/10. 1136/annrheumdis-2021-220503

6. Furer V, Eviatar T, Zisman D et al (2021) Immunogenicity and safety of the BNT162b2 mRNA COVID-19 vaccine in adult patients with autoimmune inflammatory rheumatic diseases and in the general population: a multicentre study. Ann Rheum Dis 80:1330-1338. https://doi.org/10.1136/annrheumdis-2021-220647

7. Shakoor MT, Birkenbach MP, Lynch M (2021) ANCA-associated vasculitis following Pfizer-BioNTech COVID-19 vaccine. Am J Kidney Dis 78:611-613. https://doi.org/10.1053/j.ajkd.2021.06. 016

8. McMahon DE, Amerson E, Rosenbach M et al (2021) Cutaneous reactions reported after Moderna and Pfizer COVID-19 vaccination: a registry-based study of 414 cases. J Am Acad Dermatol 85:46-55. https://doi.org/10.1016/j.jaad.2021.03.092

9. Carmona FD, Martín J, González-Gay MA (2016) New insights into the pathogenesis of giant cell arteritis and hopes for the clinic. Expert Rev Clin Immunol 12:57-66. https://doi.org/10.1586/ 1744666X.2016.1089173

10. Liozon E, Parreau S, Filloux M et al (2021) Giant cell arteritis or polymyalgia rheumatica after influenza vaccination: a study of 12 patients and a literature review. Autoimmun Rev 20:102732. https://doi.org/10.1016/j.autrev.2020.102732

Publisher's Note Springer Nature remains neutral with regard to jurisdictional claims in published maps and institutional affiliations. 